Rorsman, H., Dahlquist, I., Jacobsson, S., BrehmerAnderson, E., Ehinger, B., Linell, F. \& Rorsman, G. (1969) Tattoo granuloma and uveitis. Lancet, ii, 27.

Scadding, J.G. (1967) Sarcoidosis, p. 208. Eyre \& Spottiswood, London.
Scutr, R.W.B. (1972) The medical hazards of tattooing. Hospital Medicine, 2, 195.

Weidman, A.I., ANDrade, R. \& Franks, A.G. (1966) Sarcoidosis. Archives of Dermatology, 97, 320.

\title{
Multiple carcinomas of the large and small bowel in childhood
}

\author{
DAVID R. HARVEY \\ M.B., B.S., L.R.C.P., F.R.C.S.
}

\author{
Fernando J. Paradinas \\ L.R.C.P., L.R.C.S., M.R.C.Path.
}

\begin{abstract}
Summary
A case is reported of multiple metachronous carcinomas of the small and large intestine in a child. The latter were associated with multiple adenomatous polyps in the colon. A desmoid tumour and multiple keloids developed in the laparotomy scars. The relationship of this case to Gardner's syndrome and polyposis coli is discussed and the literature on intestinal carcinoma in childhood is briefly reviewed.
\end{abstract}

\section{Introduction}

Small bowel carcinoma has occasionally been described in association with Gardner's syndrome and large bowel carcinoma is a common late complication of this syndrome and of polyposis coli. However, we have not found previous reports of multiple small and large bowel carcinomas in this age group.

\section{Case report}

A 13-year-old girl was admitted in October 1970, with weight loss, abdominal pain, anaemia, fever and a mass in the left iliac fossa. She and two siblings had had idiopathic thrombocytopenic purpura which in her case had remitted spontaneously. At laparotomy, an ulcerating carcinoma of the lower descending colon and an adenomatous polyp found $5 \mathrm{~cm}$ distal to the carcinoma were removed by left hemicolectomy. The carcinoma was of mucoid type (Fig. 1) and involved the full thickness of the wall and one paracolic lymph node. Post-operative recovery was uneventful and extensive enquiries revealed no family history of polyposis coli or carcinoma of the colon. Follow-up barium studies revealed no further polyps.

In August 1971, she was readmitted with acute on

Reprint requests to: Dr D. R. Harvey, Charing Cross Hospital, Fulham Palace Road, London W6 8RF. subacute intestinal obstruction and a mass in the right iliac fossa. At laparotomy two small bowel carcinomas were resected: one in the upper jejunum, $7.5 \mathrm{~cm}$ distal to the duodenal jejunal flexure, and one $10 \mathrm{~cm}$ proximal to the ileocaecal junction. Microscopy showed non-mucoid adenocarcinomas involving the submucosa and inner muscle only (Fig. 2). This supported the operative impression of two separate primary small intestinal adenocarcinomas rather than metastases from the original mucoid carcinoma. Lymph nodes taken from the root of the mesentery showed marked follicular hyperplasia, but no evidence of metastases.

The patient was again admitted in April 1972 with a mass in the original colectomy scar. Clinically this was thought to be a recurrence of the carcinoma, but at operation and on subsequent histology it proved to be a desmoid tumour which was widely resected as a monobloc procedure. Reconstitution of the anterior abdominal wall was by primary suture with minimal undermining. During follow-up in 1972 repeated chest X-rays, a barium meal with follow through, a barium enema and sigmoidoscopy at 6-monthly intervals were all normal. In particular there was no evidence of further intestinal polyps.

In September 1973 the patient attended again complaining of lassitude, diarrhoea and headache. Anaemia and a mobile tumour palpable in the left iliac fossa were the clinical findings. Biopsy of a large pedunculated tumour of the rectum showed part of an adenomatous polyp. A barium enema showed multiple colonic polyps and a stricture of the sigmoid (Fig. 3). A radiological diagnosis of carcinoma was confirmed at operation. The colon showed an ulcerated stenosing tumour, merging with a sessile polyp immediately above the ulcer (Fig. 5). Up to fourteen other polyps were present in the total colectomy specimen (Figs. 4 and 5) and two further 


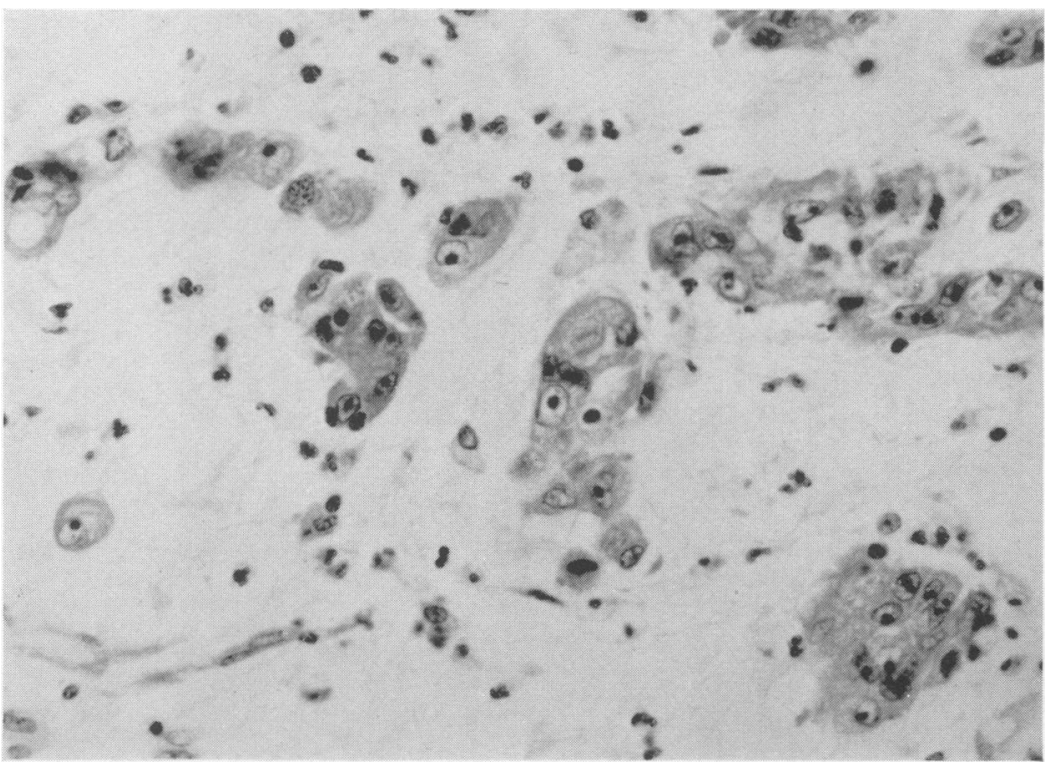

FIG. 1. Mucoid carcinoma of descending colon.

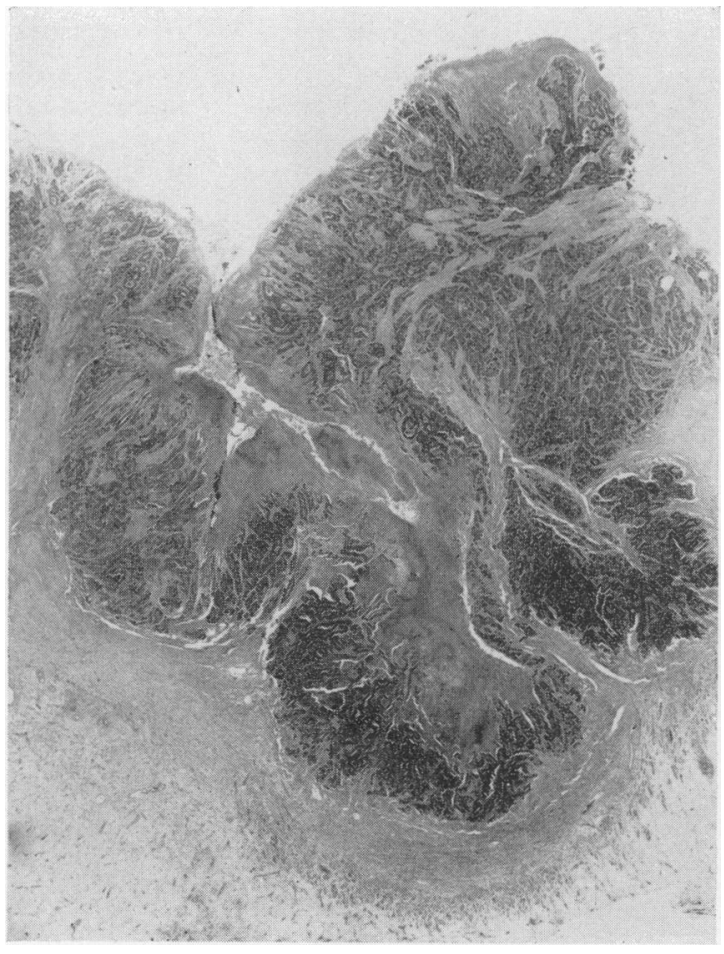

Fig. 2. Jejunal carcinoma. polyps were removed from the rectal stump after the ileorectal anastamosis had been performed. A singlệ. 기 metastasis present in the right lobe of the liver was resected.

Since then, the patient has remained well. Stool frequency settled to three soft motions per day and gains in height and weight are noticeable. Barium studies show no residual polyps in the small bowel or rectal stump and regular sigmoidoscopy to the anastomotic level remains normal. It was interesting to note that the abdominal scars have all shown marked keloid formation. Immunoglobulin estimations and lymphocyte transformation tests performed before total colectomy were within normal limits.

\section{Discussion}

In the present case, the absence of a family history of polyps and the relatively small number of polyps in the colon, suggest that the condition is not familial polyposis or polyposis coli as defined by Morson and Bussey (1970) but what these authors call 'multiple adenomatous polyps'.

Peutz-Jeghers syndrome is effectively excluded by the absence of other clinical features, the histology of the polyps, the absence of small intestinal polyps and the subsequent malignant behaviour of the colonic polyps. Despite a few reports of malignancy in Peutz-Jeghers syndrome the polyps in this syn- 


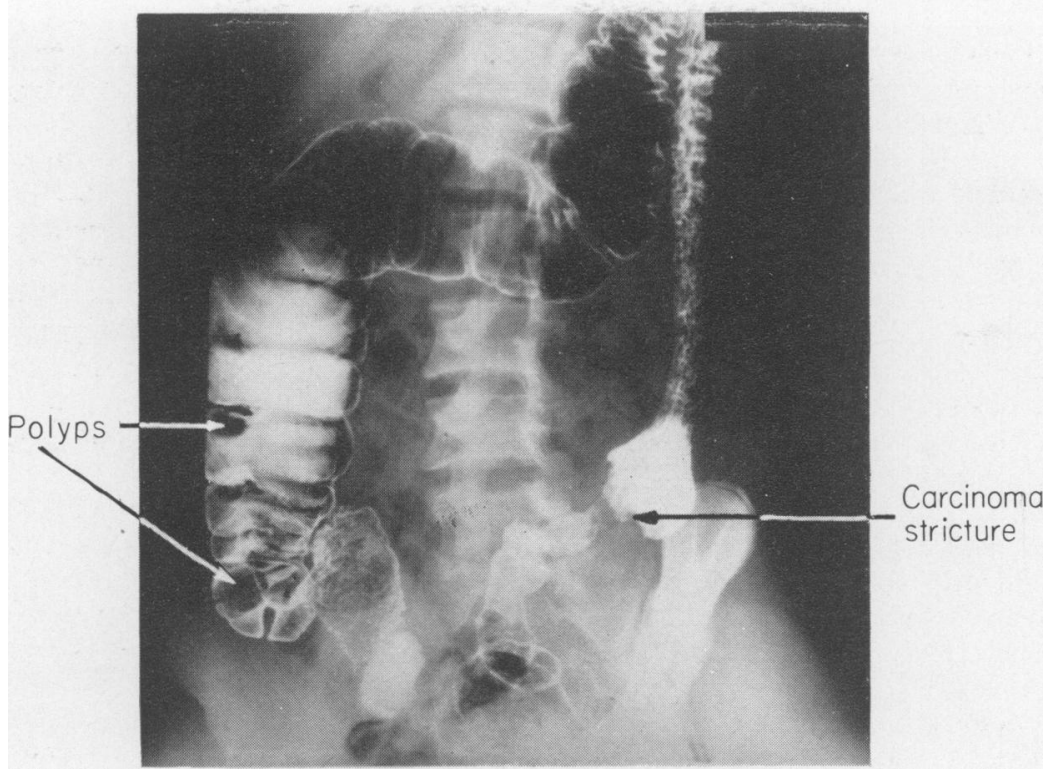

FIG. 3. Barium enema showing multiple filling defects and carcinomatous stricture.
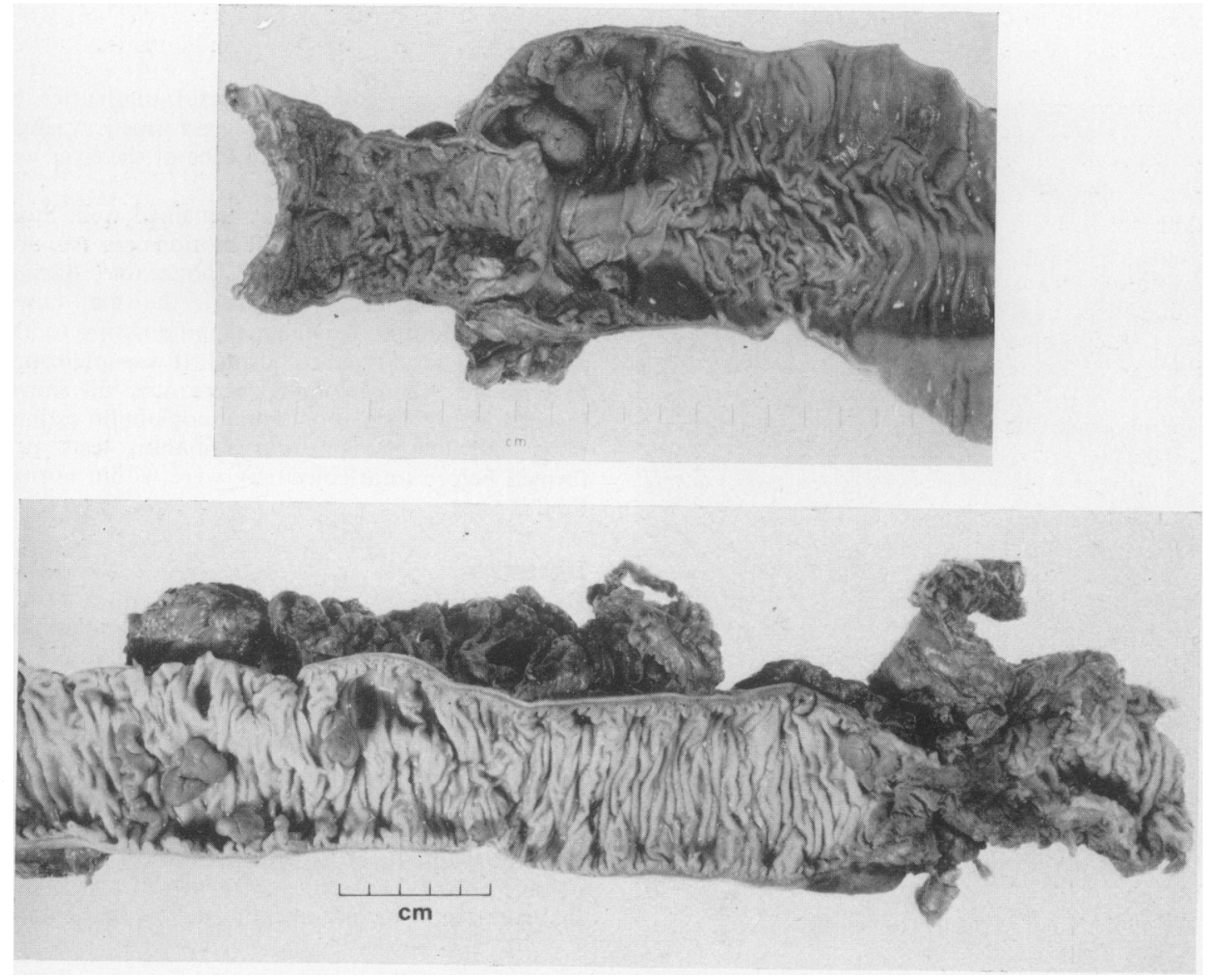

FIGs. 4 and 5. Colectomy specimen with polyps and carcinoma. 
drome are generally held to be hamartomas and not prone to carcinomatous change.

The association of large bowel adenomatous polyps with a desmoid tumour raises the possibility of Gardner's syndrome (Gardner and Richards, 1953; Gardner, 1951). However, no other features of this syndrome - epidermoid cysts, osteomas, lipomas or dentigerous cysts-were found. Desmoid tumours commonly occur after abdominal operations and have been described in this syndrome and in otherwise classical polyposis coli (Doberauer, 1972). The development of such a tumour in our patient appears to us insufficient reason to classify this case as Gardner's syndrome.

Small intestinal carcinoma is a rare tumour at any age and it is of interest that a few cases have been described in patients with Gardner's syndrome (Macdonald et al., 1967; Stalport and Letawe, 1971). In all these cases the tumour was single and located in the duodenum, whereas in our case the two tumours were in the jejunum and ileum. Bonelli (1947), in a review of the literature, found twelve reported cases of small intestinal adenocarcinoma in children; $50 \%$ of them were in the duodenum and $50 \%$ in the jejunum. No multiple carcinomas were described, but such cases have been reported in adults (Gunn, 1959).

Carcinoma of the colon in childhood is less of a rarity, but it is still uncommon, and this, together with a low index of suspicion makes the diagnosis difficult. Abdominal pain, fever, anaemia and an abdominal mass are the most usual presenting features, instead of the more commonly encountered alteration of bowel habit in adults. As in our case the diagnosis may be made with certainty only on barium studies or at laparotomy.

Carcinoma of the colon in childhood has a poor prognosis when compared with that in adults. In a series reported by Middlekamp and Haffner (1963), less than $10 \%$ of the patients had survived 5 years, the long survivals occurring in patients with well differentiated tumours. This bad prognosis may be due partly to delays in diagnosis, but also to the fact that a higher proportion of colonic carcinomas in children are of the mucoid variety (O'Brien, 1967). Mucoid carcinomas are said to metastasize early, and have a worse prognosis than other types (Wolfman, Astler and Coller, 1957). This was certainly the case in the three patients under 18 years of age reported by Cain and Longino (1970) and in other reported series in which most patients were dead with disseminated disease within 6 months (Ackerman and del Regato, 1962). The long survival of our patient after removal of a mucoid carcinoma with lymph node metastases appears to be an exception to this general rule.

It is of interest that in the present case most of the adenomatous polyps appear to have developed after the resection of the first carcinoma. In this respect there is little resemblance between this case and cases of polyposis coli and Gardner's syndrome, in which carcinomas seldom occur before the age of 20 and there is usually a long delay between the appearance of the polyps and the development of malignancy. In adults it has been noted that the presence of adenomatous polyps with a colonic carcinoma makes the presence of a second carcinoma, or its subsequent development, twice as likely (McColl, 1970). This also holds true for this particular, much younger patient.

The existence of multiple carcinomas in this case prompted investigations to assess the immunological status of the child. As far as it can be judged by the histology of the lymph nodes, the immunoglobulins and the lymphocyte transformation test, no immunological defect was present.

Finally, this case shows that recurrent symptoms $N$ and masses following the resection of carcinomas of the colon in children, as in adults, need not signify secondary spread or inoperable lesions. An active $\mathscr{D}$ surgical approach appears encouraging in the absence of widespread disease, but the long term prognosis of intestinal carcinoma in childhood must remain guarded.

\section{Acknowledgments}

We would like to thank Mr P. Stringer for his permission to publish this case, Dr R. Maini and his laboratory at the Kennedy Institute for performing the immunological competence studies and Miss $P$. Turnbull and $\mathrm{Mr}$ R. Barnet at Charing Cross Hospital for preparing the photographs.

\section{References}

ACkerman, L.V. \& del Regato, J.A. (1962) Cancer Diagnosis, Treatment and Prognosis, 3rd edn, p. 645. Mosby, St Louis.

BONELLI, W.R. (1947) Malignant tumours of the small and large intestine in infants and children. Clinical Proceedings of the Children's Hospital, Washington, 3, 151.

CAIN, A.S. \& Longino, L.A. (1970) Carcinoma of the colon in children. Journal of Pediatric Surgery, 5, 527.

Doberauer, B. (1972) Desmoid tumour and familial polyposis of the colon. Wiener Klinische Wochenschrift, 84, 32.

GARDNER, E.J. (1951) A genetic and clinical study of intestinal polyposis; a predisposing factor for carcinoma of the colon and rectum. American Journal of Human Genetics, 3, 167.

Gardner, E.J. \& Richards, R.C. (1953) Multiple cutaneous and subcutaneous lesions occurring simultaneously with hereditary polyposis and osteomatosis. American Journal of Human Genetics, 5, 139.

GuNN, S.W.A. (1959) Double carcinoma of the small intestine. Gastroenterology, 36, 256.

MCCOLL, I. (1970) Pathology and tratment of polyps of the colon and rectum. Annals of the Royal College of Surgeons of England, 47, 245.

Macdonald, J.M., Clayton Davis, W., Crago, H.R. \& BERK, A.D. (1967) Gardner's syndrome and periampullary malignancy. American Journal of Surgery, 113, 425. 
Middlekamp, J.N. \& HaffNer, H. (1963) Carcinoma of the colon in children. Pediatrics, 32, 558.

Morson, B.C. \& Bussey, H.J.R. (1970) Predisposing Causes of Intestinal Cancer-(monograph) Chicago, p. 31. Year Book Publishers, Inc.

O'BrIEN, S.E. (1967) Carcinoma of the colon in childhood and adolescence. Canadian Medical Association Journal, 96, 1217.
StalPoRT, J. \& Letawe, P. (1971) Gardner's syndrome associated with malignant degeneration of a polyp of Oddi's sphincter. Acta gastro-enterologica belgica, 34, 644.

Wolfman, E.F., Astler, V.B. \& Coller, F.A. (1957) Mucoid adenocarcinoma of colon and rectum. Surgery, St Louis, 42, 846.

\section{Familial dwarfism: case report}

\author{
S. NADER \\ B.Sc., M.R.C.P. \\ J. A. FISHER \\ M.A., M.B., B.Chir.
}

\author{
F. H. DOYLE \\ F.F.R. \\ K. MASHiter \\ Ph.D. \\ G. F. JOPLIN \\ Ph.D., F.R.C.P.
}

\begin{abstract}
Summary
Three sisters are described, of consanguinous parents, with multiple trophic hormone deficiencies presenting as short stature. In two of them the size of the sella turcica is large in relation to the skeletal age and height. All three show an acute metabolic response to an administered preparation of human growth hormone.
\end{abstract}

\section{Introduction}

Growth hormone deficiency resulting in short stature may occur in the absence of an obvious structural lesion in the pituitary-hypothalamic area as an isolated defect or as part of the syndrome of idiopathic hypopituitarism. Both may occur sporadically or in families (Goodman, Grumbach and Kaplan, 1968). Studies of families suggest autosomal recessive inheritance (Trygstad and Seip, 1964). Until recently it was only possible to guess that trophic hormone deficiency existed by extrapolating from tests of pituitary function but with the availability of gonadotrophin releasing hormone (LH/FSH-RH) and thyrotrophin releasing hormone (TRH) and serum assays for pituitary hormones defective pituitary reserve can be documented. Job et al. (1972) showed a failure of thyrotrophin (TSH) to rise in response to TRH in a dwarfed child who had growth hormone deficiency and Chaussain et al. (1972) have tested gonadotrophin reserve in similar children. In their series of five patients with growth hormone deficiency, one female had a normal gonadotrophin reserve and four males had impaired or absent reserves.

Radiological studies have shown that the pituitary fossa size of one-third of patients with idiopathic hypopituitarism is abnormally small, the volume being more than two standard deviations below the age and height related means of the control group (Underwood et al., 1973). This is in keeping with the findings of Goodman et al. (1968) and our own experience.

The nitrogen retaining and hypercalcuric action of growth hormone can be used to test growth hormone responsiveness in susceptible subjects. Though there are conflicting reports as to prognostic use as far as future growth is concerned (Melvin et al., 1967; Clayton, Tanner and Vince, 1971) these tests are of value in demonstrating target organ sensitivity to an administered preparation of human growth hormone.

We report on three sisters, of consanguinous healthy parents, with multiple trophic hormone deficiencies presenting as short stature. Our data includes assessment of their pituitary function, their acute response to human growth hormone and we 